\title{
Bacteraemia and Antibiotic Susceptibility Pattern of Isolates from Patients Visiting a Private Hospital of Kathmandu
}

\author{
Nandalal Jaishi ${ }^{1}$, Pramila Pathak ${ }^{2}$ Pradeep Kumar Shah ${ }^{2}$, Puspa Raj Dahal ${ }^{1 *}$ \\ ${ }^{1}$ Department of Microbiology, Tri-Chandra Multiple Campus, Ghantaghar, Kathmandu, \\ * Corresponding author: Puspa Raj Dahal; Department of Microbiology, Tri-Chandra Multiple Campus, \\ Ghantaghar, Kathmandu; E-mail: pusparajdahal@gmail.com
}

\begin{abstract}
Objectives: This study was conducted to identify the causative agents of bacteraemia and to assess antibiogram of the isolates among the patients suspected of blood stream infection visiting Everest hospital, New Baneshwor Kathmandu.
\end{abstract}

Methods: Altogether 400 blood cultures were processed during March to August, 2015. Standard Operating Procedures (SOPs) was followed during the processing of the specimens. Antibiotic susceptibility testing of bacterial isolates was done by Kirby Bauer disc diffusion method with MullerHinton agar using the guidelines and interpretive criteria of the Clinical and Laboratory Standards Institute (CLSI 2013).

Results: The positivity of blood culture was found to be $48(12 \%)$. Gram negative bacteria were found to be more predominant $27(56.2 \%)$ than Gram positive bacteria $21(43.7 \%)$ in causing bacteraemia. The most prevalent isolate was Staphylococcus aureus 15 (31.2\%) followed by Salmonella Paratyphi A $10(20.8 \%)$ and Salmonella Typhi 8 (16.6\%), Escherichia coli \& CoNS 4 (8.3\%), Pseudomonas aeruginosa 3 (6.2\%) and Klebsiella pneumoniae and Streptococcus pneumoniae 2 (4.1\%) respectively. All Gram-positive isolates were found to be sensitive to Cefoxitin, Ceftriaxone and Vancomycin followed by Ampicillin (90.42\%), Erythromycin (85.71\%), Ciprofloxacin (83.33\%), Doxycycline (75\%) and Cephalexin $(70.58 \%)$ whereas Gram negative isolates were sensitive to Ceftriaxone followed by Chloramphenicol (92\%), Gentamicin (88.8\%), Cefixime (85.71\%), Ofloxacin (83.3\%) and Amoxycillin and Ciprofloxacin $(71.3 \%)$.

Conclusion: The isolation of etiological agents of blood stream infection should be assessed by proper microbiological analysis and it would be helpful for controlling of the outbreaks of resistance strains through effective empirical therapy.

Key words: Non-fermentative gram-negative bacteria (NFGNB), bloodstream infections, BHI

\section{INTRODUCTION}

Continuous or transient presence of bacteria with in the blood stream is bacteraemia which may indicate an intravascular infection, pneumonia or liver abscess (Forbes, et al., 2007). It is the most common cause of significant morbidity that leads to mortality especially in the developing countries ( Karlowsky, et al., 2004), (Pittet, et al., 1994) despite the availability of potent antimicrobial therapy and advances in supportive care. Both gram positive and gram negative bacteria causes bacteraemia and septicemia. Gram negative

Date of Submission: August 20, 2018

Published Online: January 2019 septicemia, also known as endotoxic shock, is more severe than gram positive septicemia. Staphylococcus aureus, Escherichia coli, coagulase-negative staphylococci (CoNS), Klebsiella pneumoniae, Pseudomonas aeruginosa, Enterococcus spp., Streptococcus spp., Candida albicans, and Enterobacter cloacae are the most frequent etiological agents of bacteraemia and fungaemia in Europe and the United States (Cleven, et al., 2006). Infections caused by non-fermentative gram-negative bacteria (NFGNB) is a significant problem in both hospitalized and community-dwelling patients due to their ubiquitous

Date of Acceptance: November 18, 2018

DOI: https://doi.org/10.3126/tujm.v5i0.22300 
distributions in the environment and increasing incidence of multidrug resistance (Enoch, et al., 2007), (Suárez et al. 2005). Gram-negative bacillary sepsis with shock has a mortality rate of 12 to 38 percent; mortality varies depending, in part, on whether the patient receives timely and appropriate antibiotic therapy (Kang et al. 2005), (Kumar et al. 2006). Mortality appears to be higher with methicillin-resistant (MRSA) compared with methicillin-sensitive S. aureus (MSSA) bacteremia (Mylotte et al. 1987). Effective treatment of gram-positive bloodstream infections including those caused by Staphylococcus, Streptococcus and Enterococcus species, represents major clinical challenges (Christoph et al. 2009). This is necessary intervention program to monitor the spread of resistant bacteria and their antibiogram with the aim to identify the common bacterial agents involved in BSIs in Nepal. This would help health professionals technicians to know about the current situation of bacteraemia.

\section{MATERIALS AND METHODS}

The study was conducted on 400 blood samples collected during March, 2015 to August, 2015 from male and female patients of all age groups, who were suspected of bacteraemia by the clinicians attending Everest Hospital, New Baneshwor, Kathmandu, Nepal.

All blood samples, for culture $3 \mathrm{ml}$ from children and $5 \mathrm{ml}$ from adult were collected from peripheral vein aseptically before starting antibiotic therapy and inoculated into $45 \mathrm{ml}$ of sterile brain heart infusion broth supplemented with Sodium Polyanethol Sulphonate (SPS). Inoculated medium was thoroughly mixed to avoid clotting of the blood and immediately transported to the microbiology laboratory for further procedures. BHI broth was incubated at $37^{\circ} \mathrm{C}$ upto 7 days unless the visible growth was obtained. The culture bottles were examined daily for visual evidence of microbial growth such as haemolysis, turbidity, gas production, pellicle formation and "puffballs" and clotting which indicates the positive microbial growth. Subcultures were performed from positive culture bottles on blood agar and MacConkey agar. Plates were incubated at $37^{\circ} \mathrm{C}$ for 24 hours (Forbes, et al., 2007). The identification of bacteria from isolated colonies was done by standard microbiological procedures as described in Bergey's Manual (second edition 2004), which involve colony morphology, Gram stain and biochemical reaction. Antibiotic susceptibility testing of bacterial isolates was done by Kirby Bauer disc diffusion method with Muller-Hinton agar using the guidelines and interpretive criteria of the Clinical and Laboratory Standards Institute (CLSI 2013). Control strains of E. coli (ATCC 25922) and Staphylococcus aureus (ATCC 25923) were used for the standardization of the Kirby-Bauer test and also for correct interpretation of zone of diameter. Statistical analysis was done by using SPSS version 16. Frequency and percentages were calculated and Chi-square test was done whenever applicable with $\mathrm{P}<0.05$ regarded as significant.

\section{RESULTS}

Out of total 400 blood samples processed for culture, $48(12 \%)$ showed bacterial growth whereas 352 (88\%) showed no growth. Among 48 culture positive isolates, $81.2 \%$ were from outpatients and remaining $18.7 \%$ were from inpatients. Out of total bacterial isolates $(n=48)$, Gram negative bacteria were found to be 27 (56.2\%) with Salmonella Typhi, Paratyphi A, E. coli, pseudomonas aeruginosa and Klebsiella pneumoniae being the common microbes. Out of $21(43.7 \%)$ gram positive bacteria, Staphylococcus aureus (31.2\%) was found to be most predominance followed by CoNS and Streptococcus pneumoniae.

Table 1: List of bacterial isolates

\begin{tabular}{lcc}
\hline Types of bacteria & Number & Percentage (N=48) \\
\hline Gram Negative Bacteria & 27 & 56.2 \\
\hline Salmonella Typhi & 8 & 16.6 \\
Salmonella Paratyphi A & 10 & 20.8 \\
Escherichia coli & 4 & 8.3 \\
Pseudomonas aeruginosa & 3 & 6.2 \\
Klebsiella pneumoniae & 2 & 4.1 \\
\hline Gram positive bacteria & 21 & 43.7 \\
\hline Staphylococcus aureus & 15 & 31.2 \\
CoNS & 4 & 8.3 \\
Streptococcus pneumoniae & 2 & 4.1 \\
\hline
\end{tabular}


Table 2: Distribution pattern of the bacterial isolates in blood cultures of different age group

\begin{tabular}{lcccccccccc}
\hline \multirow{2}{*}{ Types of organism isolated } & \multicolumn{7}{c}{ Age group (years) } & \multirow{2}{*}{ Total } \\
\cline { 2 - 9 } & $\mathbf{0 - 1 0}$ & $\mathbf{1 1 - 2 0}$ & $\mathbf{2 1 - 3 0}$ & $\mathbf{3 1 - 4 0}$ & $\mathbf{4 1 - 5 0}$ & $\mathbf{5 1 - 6 0}$ & $\mathbf{6 1 - 7 0}$ & $>\mathbf{7 1}$ & $\mathbf{8}$ \\
\hline Salmonella Typhi & 4 & 0 & 1 & 0 & 2 & 0 & 1 & 0 & 1 \\
Salmonella Paratyphi A & 2 & 1 & 2 & 3 & 0 & 1 & 0 & 1 & 10 \\
Escherichia coli & 0 & 1 & 2 & 0 & 0 & 1 & 0 & 0 & 4 \\
Pseudomonas aeruginosa & 0 & 0 & 1 & 2 & 0 & 0 & 0 & 0 & 3 \\
Klebsiella pneumoniae & 2 & 0 & 0 & 0 & 0 & 0 & 0 & 0 & 2 \\
Staphylococcus aureus & 1 & 2 & 1 & 4 & 2 & 5 & 0 & 0 & 15 \\
CoNS & 0 & 0 & 2 & 1 & 0 & 1 & 0 & 0 & 4 \\
Streptococcus pneumoniae & 2 & 0 & 0 & 0 & 0 & 0 & 0 & 0 & 2 \\
\hline Total (No.) & 11 & 4 & 9 & 10 & 4 & 8 & 1 & 1 & 48 \\
\hline
\end{tabular}

Among the total patients, $262(65.5 \%)$ were male showed 31/262 (11.8\%) growth and remaining 138 (34.5\%) female showed 17/138 (12.3\%) growth. The age group between 21 to 30 years had the maximum requests of $110(27.5 \%)$ for blood culture followed by age group between 11 to 20 years 89 (22.2\%) whereas, age group above 71 years found to be least with 15 $(3.7 \%)$ requests.
All gram-positive isolates were found to be sensitive to Cefoxitin, Ceftriaxone and Vancomycin followed by Ampicillin (90.4\%), Erythromycin (85.7\%), Ciprofloxacin (83.3\%), Doxycycline (75\%) and Cephalexin (70.5\%) whereas Gram negative isolates were sensitive to Ceftriaxone followed by Chloramphenicol (92\%), Gentamicin (88.8\%), Cefixime (85.7\%), Ofloxacin (83.3\%) and Amoxycillin and Ciprofloxacin (71.4\%) (Table 3 and Table 4).

Table 3: Antibiotic susceptibility pattern of Gram negative bacteria

\begin{tabular}{lccc}
\hline \multirow{2}{*}{ Antibiotics } & Total no. of isolates & \multicolumn{2}{c}{ Susceptibility pattern } \\
\cline { 3 - 4 } & 27 & Resistant (\%) & Susceptible (\%) \\
\hline Amoxycillin & 17 & $6(28.5)$ & $21(71.4)$ \\
Cotrimoxazole & 14 & $6(35.2)$ & $11(64.7)$ \\
Cefixime & 24 & $2(14.2)$ & $12(85.7)$ \\
Ofloxacin & 27 & $4(16.6)$ & $20(83.3)$ \\
Ciprofloxacin & 23 & $6(28.5)$ & $21(71.4)$ \\
Ceftriaxone & 25 & $0(0)$ & $23(100)$ \\
Chloramphenicol & 18 & $2(8)$ & $23(92)$ \\
Nalidixic acid & 9 & $12(66.6)$ & $6(33.3)$ \\
Gentamicin & 15 & $1(11.1)$ & $8(88.8)$ \\
Cefotaxime & $5(33.3)$ & $10(66.6)$ \\
\hline
\end{tabular}

Table 4: Antibiotic susceptibility pattern of Gram positive bacteria

\begin{tabular}{lccc}
\hline \multirow{2}{*}{ Antibiotics } & Total no. of isolates & \multicolumn{2}{c}{ Susceptibility pattern } \\
\cline { 3 - 4 } & & $2(9.5)$ & Susceptible (\%) \\
\hline Ampicillin & 21 & $12(57.1)$ & $9(90.4)$ \\
Cotrimoxazole & 21 & $0(0)$ & $4(100)$ \\
Chloramphenicol & 4 & $1(16.6)$ & $5(83.3)$ \\
Ciprofloxacin & 6 & $0(0)$ & $19(100)$ \\
Cefoxitin & 19 & $0(0)$ & $17(100)$ \\
Ceftriaxone & 17 & $1(25)$ & $3(75)$ \\
Doxycycline & 4 & $0(0)$ & $19(100)$ \\
Vancomycin & 19 & $3(14.2)$ & $18(85.7)$ \\
Erythromycin & 21 & $5(29.4)$ & $12(70.5)$ \\
Cephalexin & 17 & $0(0)$ & $15(15)$ \\
Clindamycin & 15 & & \\
\hline
\end{tabular}




\section{DISCUSSION}

The present study was conducted to isolate the bacteria causing blood stream infection and their antimicrobial susceptibility pattern among the patient visiting Everest hospital, New Baneshwor Kathmandu. The isolation rate of blood culture positive cases was $12 \%$ which was similar to the study conducted by (Pandey et al. 2013) reporting a $12.6 \%$ blood culture positivity rate and a study from western Nepal in 2007 with isolation rate 10.2\% (Easow et al. 2010), while a similar study which was done in Iran also showed a lower positivity rate of 5.6\% (Mehdinejad et al. 2009). A very recent study done in south India also showed $8.3 \%$ culture positive samples (Vanitha et al. 2012) whereas (Meremikwu et al. 2005) reported $45.9 \%$ of positive blood culture. The variation in the blood culture positivity may be attributed to the factors like number and amount of blood culture taken for screen (Lee et al. 2007).

Gram negative bacteria $(56.25 \%)$ were found to be predominant in our study which was similar as reported by (Osinupebi \& Olajubu 2003) and (Sharma et al. 2006). The predominance of Gram-negative organisms reported as etiological agents of bacteraemia and septicaemia was also seen in the study done by (Mehta et al. 2005) where Gram-negative bacteria accounted for $80.96 \%$. This is in contrast to the study by (Babay et al. 2005) revealed the predominance of gram positive bacteria (78.6\%). And also, from finding by (Chaudhry et al. 2000); (Khanal et al. 2007), (Lee et al. 2007), Gram positive bacteria as the predominant. Variation of such etiology are due to changes in geographical location and antibiotic policy advocated in hospital which reflects the better isolation of patients in hospital and hand washing practices in ICU or high-risk unit in hospital (Mahmood 2001). S. aureus 31.2\% (15/48) was isolated as the most common causative agent followed by S. Paratyphi A $20.8 \%$ (10/48), Salmonella Typhi $16.6 \%$ (8/48), Klebsiella pneumoniae $6.2 \%$ (3/48), P. aeruginosa $6.2 \%(3 / 48)$, E. coli $8.3 \%(4 / 48)$, Streptococcus pneumoniae $4.1 \%$ (2/48) and CoNS 6.2\% (3/48). Similar finding reported by Swain and Otto, 2012, where they reported Staphylococcus was common organism (73\%).

In this study, analysis of blood samples covers almost all aged patients in which the highest frequency of infection was in the age group (0-10) years that was (22.9\%). Among the positive blood culture male was $11.8 \%(31 / 262)$ and the female was $12.3 \%(17 / 138)$ Similar finding was reported by (Manandhar et al.
2009), in their study conducted at Sahid Gangalal National Heart Centre, Bansbari, Nepal and studies from both Nepal and other parts of the world (Kandel et al. 2007); (Mylonakis \& Calderwood, 2001). Physical stress, smoking habits and types of invasive procedures could contribute for impairments of cardiac conditions leading to bacteremia. Among the total population, the highest culture positive was from outpatients $81.2 \%$ which might be because the patients were in the initial phase of infection without undergone any antibiotic therapy. The highest peak of culture positivity was seen in the month of March 2015 followed by August, May, July, June and April. This may be possibly due to sewage mediated contamination of drinking water (Rai et al. 2005). Another factor influencing this occurrence might be scanty and contaminated community water supply in summer and rainy season (Bhatta et al. 2007).

In our study, gram-positive isolates showed $100 \%$ sensitivetowardsCefoxitin, CeftriaxoneandVancomycin followed by Ampicillin (90.4\%), Erythromycin $(85.7 \%)$, Ciprofloxacin (83.3\%), Doxycycline (75\%) and Cephalexin $(70.5 \%)$ but showed resistance towards Cotrimoxazole similar pattern was reported by (Lee et al. 2007) whose found shows that gram positive isolates was sensitive to most of the antibiotics including Ampicillin, penicillin and vancomycin. Similarly, gram negative isolates showed $100 \%$ sensitive to Ceftriaxone followed by Chloramphenicol (92\%), Gentamicin (88.8\%), Cefixime (85.7\%), Ofloxacin (83.3\%) and Amoxycillin and Ciprofloxacin (71.4\%). The antibiotic Nalidixic acid (66.6\%) was found least effective towards Gram negative isolates resembling with the study conducted by (Tibrewal, 1999) found that ciprofloxacin was the most effective antibiotic (93.2\%) whereas ampicillin was found to be $20.2 \%$ effective against all the 89 gram-negative isolates. It has been suggested that resistance to nalidixic acid may be an indicator of low-level resistance to ciprofloxacin (Vasallo et al. 1998).

\section{CONCLUSION}

Bacteraemia is emerging as an important disease in our community. Proper identification of isolates using microbiological tools should be undertaken.

\section{ACKNOWLEDGEMENTS}

We would like to thank all the patients whose samples were used in the study, all the staff of Everest Hospital, 
New Baneshwor, Kathmandu, all the faculties and staff of Department of Microbiology, Tri-Chandra Multiple Campus, and all my friends and family members.

\section{CONFLICT OF INTEREST}

The authors declare no conflict of interest.

\section{REFERENCES}

Karlowsky JA et al. (2004). Prevalence and antimicrobial susceptibilities of bacteria isolated from blood cultures of hospitalized patients in the United States in 2002. Ann Clin Microbiol Antimicrob, 3: 7.

Babay HA, Twum-Danso K, Kambal AM \& Al-Otaibi FE (2005). Bloodstream infections in pediatric patients. Saudi Med J 26: 1555-1561.

Bhatta DR et al. (2007). Serotyping, PCR, phage-typing and antibiotic sensitivity testing of Salmonella serovars isolated from urban drinking water supply systems of Nepal. App Microbial Newsletter 44: 588-94.

Chaudhry I et al. (2000). Etiologcal pattern of septicemia at three Hospitals in Lahore. JCPSP 10: 375-379.

Christoph KN et al. (2009). Clinical Consensus Conference: Survey on Gram-Positive Bloodstream Infections with a Focus on Staphylococcus aureus. Clin Infect Dis 48(Suppl 4): S260-S270.

Cleven BE et al. (2006). Identification and characterization of bacterial pathogens causing bloodstream infections by DNA microarray. J Clin Microbiol 44(7): 2389-97.

Easow MJ et al. (2010). Blood Stream Infections among febrile patients attending a teaching hospital in Westren Region of Nepal. AJM, 3(10): 633-37.

Enoch DA, Birkett CI and Ludlam HA (2007). Nonfermentative Gram-negative bacteria. Int $J$ Antimicrob Agents,29(Suppl 3): S33-S41.

Forbes BA, Sahm DF \& Weissfeld AS (2007). Bailey $\mathcal{E}$ Scott's Diagnostic Microbiology.12th ed. s.1.:Mosby.

Kandel NP et al. (2007). Laboratory tests for infective endocarditis among patients visiting Sahid Gangalal National Heart Centre, Bansbari, Kathmandu, Nepal. J Nepal Health Res Counc 1: 5.

Kang CI et al. (2005). Bloodstream infections caused by antibiotic-resistant gram-negative bacilli: risk factors for mortality and impact of inappropriate initial antimicrobial therapy on outcome. Antimicrob Agents Chemother 49(2): 760-6.

Khanal B et al. (2007). Antimicrobial susceptibility patterns of Salmonella enterica serotype Typhi in Eastern Nepal. J H Pop Nutr 25: 28-7.

Kumar A et al. (2006). Duration of hypotension before initiation of effective antimicrobial therapy is the critical determinant of survival in human septic shock. Crit Care Med 36(6): 1589-96.

Lee A, Mirrett S, Reller LB and Weinstein MP (2007). Detection of bloodstream infections in adults: how many blood cultures are needed?. J Clin. Microbial 45(11): 3546-8.

Mahmood A (2001). Blood stream infections in a medical intensive care unit: spectrum and antibiotic susceptibility pattern. J Pak Med Assoc 51: 213-15.

Manandhar S et al. (2009). Bacteriological profile of heart valves resected from infective endocarditis patients. J Nepal Health Res Counc 7: 108-11.

Mehdinejad A, Khosravi D and Morvaridi A (2009). Study of prevalence and antimicrobial susceptibility pattern of bacteria isolated from blood cultures. J Bio Sci 249-53.

Mehta M, Dutta P \& Gupta V (2005). Antimicrobial susceptibility pattern of blood isolates from a teaching hospital in north India. Jpn J Infect Dis 58: $174-76$.

Meremikwu MM et al. (2005). Bacterial isolates from blood cultures of children with suspected septicaemia in Calabar, Nigeria. BMC Infect Dis 5:110.

Mylonakis E and Calderwood SB (2001). Infective endocarditis in adults. N Engl J Med 1318-30.

Mylotte JM, McDermott C and Spooner JA (1987). Prospective study of 114 consecutive episodes of Staphylococcus aureus bacteremia. Rev Infect Dis 9(5): 891-907.

Osinupebi OA and Olajubu FA (2003). Afr J Med Med Sci Volume 32: 311-14.

Pandey S, Raza S and Bhatta CP (2013). The Aetiology of the Bloodstream Infections in the Patients Who Presented to a Tertiary Care Teaching Hospital in 
Kathmandu, Nepal. J Clin Diagn Res 7(4): 638-41.

Pittet D, Tarara D and Wenzel RP (1994). Nosocomial bloodstream infection in critically ill patients. Excess length of stay, extra costs, and attributable mortality. JAMA 271(20): 1598-601.

Rai DR, Kshetry NT, Tamang MD and Pokharel BM (2005). Study of enteric fever and malaria incidence in southern part of Nepal. J Inst Med 27(3): 46-51.

Sharma NP et al. (2006). A hospital-based study of bloodstream infections in febrile patients in Dhulikhel Hospital Kathmandu University Teaching Hospital, Nepal. Southeast Asian J Trop Med Public Health 37: 315-56.

Suárez CJ, Lolans K, Villegas MV and Quinn JP (2005). Mechanisms of resistance to $\beta$-lactams in some common Gram-negative bacteria causing nosocomial infections. Expert Rev Anti Infect Ther
3(6): 915-922

Tibrewal A (1999). A Prospective Study on Etiological Agents Causing Infective Endocarditis and Related Bacteraemic and Septicaemic Cases among Patients Visiting Bir Hospital. M.Sc. Dissertation, Submitted to Central Department of Microbiology, Tribhuvan University, Kathmandu.

Vanitha RN et al. (2012). A retrospective study on blood stream infections and antibiotic susceptibility patterns in a tertiary care teaching hospital. Int $J$ Pharm Pharm Sci 4: 543-48.

Vasallo FJ et al. (1998). Failure of ciprofloxacin therapy for invasive nontyphoidal salmonellosis. Clin Infect Dis, 26: 335-36.

Wayne P (2003). Performances, Standards for Antimicrobial Disk Susceptibility. PA 19087 USA. 